\title{
On the Time-Dependent Behavior of FGM Plates
}

\author{
Holm Altenbach ${ }^{1, a}$ and Victor A. Eremeyev ${ }^{2, b}$ \\ ${ }^{1}$ Department of Engineering Sciences, Martin-Luther-University Halle-Wittenberg, D-06099, Halle, \\ Germany \\ ${ }^{2}$ South Scientific Center of RASci \& South Federal University, Milchakova St. 8a, 344090 Rostov \\ on Don, Russian Federation \\ a)holm.altenbach@iw.uni-halle.de, b) eremeyev.victor@gmail.com
}

Keywords: Time-dependent behavior, Functionally graded materials, Plates, Viscoelasticity

Abstract. A non-classical plate theory based on the direct approach is introduced and applied to plates composed of functionally graded materials (FGM). The governing two-dimensional equations are formulated for a deformable surface, the viscoelastic stiffness parameters are identified assuming linear-viscoelastic material behavior. In addition, the material properties are changing in the thickness direction. Solving some problems of the global structural analysis it can be shown that in some cases the results based on the presented theory significantly differ from the results based on the Kirchhofftype theory.

\section{Introduction}

Functionally graded materials are a very perspective class of materials for modern engineering applications. In contrast to classical structural materials like steel or plastics they are macroscopically inhomogeneous. In this sense foams belong to the FGMs. Metallic and polymeric foams are more and more used as a material for lightweight structures [1, 2, 3, 4, 5, 6, 7]. Such structures are applied in civil engineering, in the automotive or airspace industries since they combine low weight, high specific strength, and excellent possibilities to absorb energy. The technical realization is mostly performed as sandwich panels (plates or shells with hard and stiff face sheets and a core layer made of a foam). From the mechanical point of view, a foam is a very complex material which has many non-classical properties (for example, some foams have a negative Poisson's ratio, see $[4,8,9]$ for details). The non-homogeneous foam itself can be modeled as a functionally graded material (FGM) with "smeared" mechanical properties changing over the thickness direction. There are two types of metal or polymer foams. One is the closed-cell foam, while the second one is the open-cell foam. The defining property of the foams is the very high porosity: typically well over $80 \%, 90 \%$ and even $98 \%$ of the volume consists of void spaces. Examples of polymer open-cell foam and the foam with non-homogeneous porosity are presented in Fig. 1.

The analysis of plate and shell structures can be performed on the base of theories deduced by various approaches. Finally, all theories are presented by two-dimensional field equations. Mostly engineering hypotheses about the stress, the strain or the displacement states in the plate are applied, and one gets some simplifications of the basic three-dimensional equations. They result in two-dimensional equations connecting the kinematics with the strains, stating the static or dynamic equilibria and founding the constitutive relations. In a similar way applying some mathematical techniques like the power series expansion or the asymptotic integration one can observe the set of two-dimensional governing equations. Note that both approaches have a unique starting point the equations of the three-dimensional continuum. In contrast, the direct approach is based on the straight-forward introduction of two-dimensional equations without any a priori three-dimensional assumptions. This approach in combination with the effective properties concept allows the global 

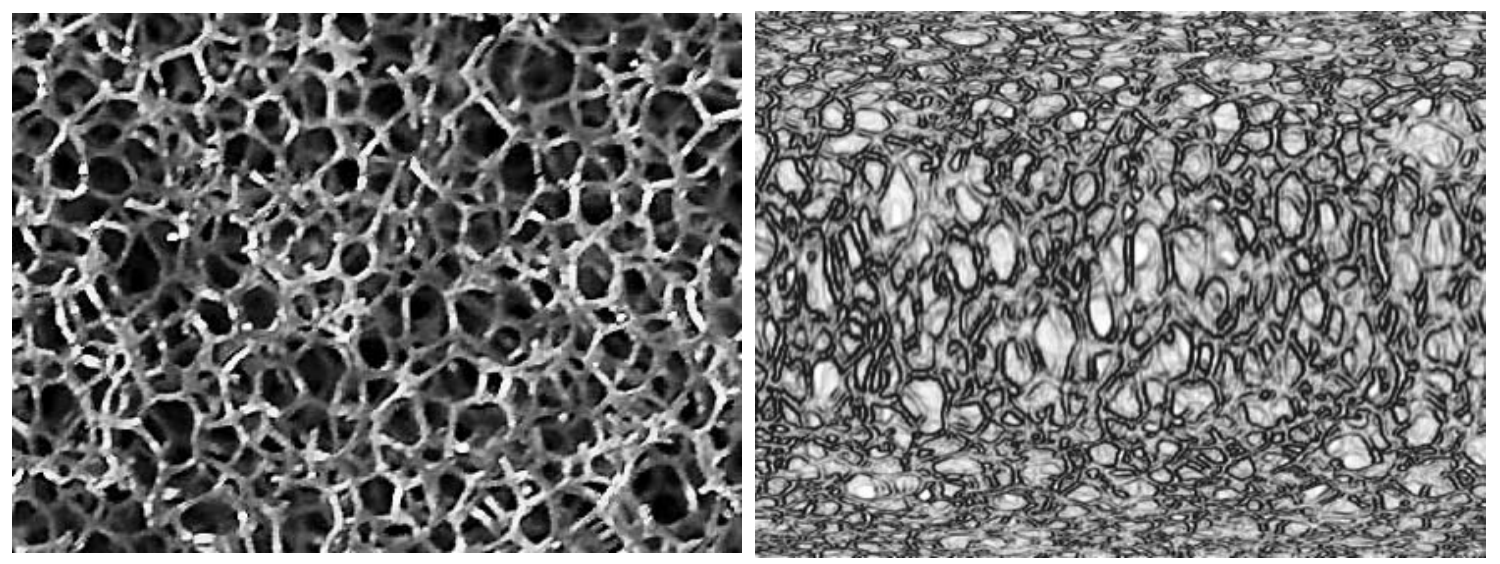

Fig. 1. Polymer open-cell (left) foam and nonhomogeneous structures (right)

structural analysis in all branches of the plate theory (homogeneous, sandwich, laminated, etc.). The various possibilities of the formulation of plate theories are discussed in $[10,11]$ among others.

Here we present a non-classical theory of viscoelastic plates with changing properties in the thickness direction based on the direct approach in the plate theory and added by the effective properties concept. We consider plates made of polymer foams with a highly nonhomogeneous structure through the thickness (see, for example Fig. 1). The theory of plates which will be applied was formulated earlier in $[12,13,14,15,16]$. A similar approach was suggested in [17], but the homogeneous material behavior was assumed. From the direct approach point of view the plate is modeled as a material surface each point of which has five degrees of freedom (three displacements and two rotations, the rotation about the normal to the plate surface is not considered as a kinematically independent variable). Such a model can be accepted in the case of plates with constant or slow changing thickness. For the linear variant the identification of the elastic and viscoelastic stiffness tensors considering changing properties was proposed in $[18,19,20]$. Using the techniques presented in these articles the static boundary-value problems for FGM plates made of metal foams which behave elastically are solved in [21].

\section{Governing Equations}

Let us consider for the brevity the geometrically and physically linear theory. The basic equations interlinking the strains with the displacements and the rotations or stating the equilibrium can be deduced applying hypotheses (e.g., the Kirchhoff's hypotheses or the Reissner's hypotheses) or mathematical techniques (like power series expansion or asymptotic integration). In both cases one gets automatically the expressions for the constitutive equations assuming elastic or inelastic material behavior.

A quite different way is given by the direct approach. The starting point in this case is a twodimensional deformable surface. On each part of this deformable surface forces and moments are acting - they are the primary variables. The next step is the introduction of the deformation measures. Finally, it is necessary to formulate constitutive equations. In comparison with the other approaches such a theory is formulated in a more natural way. But the identification of the effective properties (e.g., stiffness parameters) must be realized for each class of plates individually solving boundary value or eigen-vibration problems. The identification of the two-dimensional characteristics is a nontrivial problem since they must be computed from the three-dimensional material parameters applying assumptions like the introduction of stress resultants (forces and moments) instead of the stress tensor components.

Let us introduce the governing equations. The equations of motion are formulated as the Euler's 
laws of dynamics [19, 20, 21]

$$
\nabla \cdot T+\boldsymbol{q}=\rho \ddot{u}+\rho \boldsymbol{\Theta}_{1} \cdot \ddot{\varphi}, \quad \nabla \cdot \boldsymbol{M}+T_{\times}+\boldsymbol{m}=\rho \boldsymbol{\Theta}_{1}^{\mathrm{T}} \cdot \ddot{u}+\rho \boldsymbol{\Theta}_{2} \cdot \ddot{\boldsymbol{\varphi}} .
$$

Here $\boldsymbol{T}, \boldsymbol{M}$ are the tensors of forces and moments, $\boldsymbol{q}, \boldsymbol{m}$ are the vectors of surface loads (forces and moments), $T_{\times}$is the vector invariant of the force tensor, $\nabla$ is the nabla (Hamilton) operator, $\boldsymbol{u}, \boldsymbol{\varphi}$ are the vectors of displacements and rotations, $\Theta_{1}, \Theta_{2}$ are the first and the second tensor of inertia, $\rho$ is the density (effective property of the deformable surface), $(\ldots)^{\mathrm{T}}$ denotes transposed and $(\ldots)$ the time derivative. The geometrical equations are given as

$$
\mu=(\nabla u \cdot a)^{\mathrm{sym}}, \quad \gamma=\nabla u \cdot n+c \cdot \varphi, \quad \kappa=\nabla \varphi .
$$

$a$ is the first metric tensor (plane tensor), $n$ is the unit outer normal vector at the surface, $c$ is the discriminant tensor $(c=-a \times n), \mu, \gamma$ and $\kappa$ are the strain tensors (the tensor of in-plane strains, the vector of transverse shear strains and the tensor of the out-of-plane strains), (...) sym denotes the symmetric part.

The boundary conditions are given by the relations

$$
v \cdot T=f, \quad v \cdot M=l \quad(\boldsymbol{l} \cdot \boldsymbol{n}=0) \quad \text { or } \quad \boldsymbol{u}=\boldsymbol{u}^{0}, \quad \boldsymbol{\varphi}=\boldsymbol{\varphi}^{0} \quad \text { along } S .
$$

Here $f$ and $\boldsymbol{l}$ are the external force and moment vectors acting along the boundary $S$ of the plate, while $\boldsymbol{u}^{0}$ and $\boldsymbol{\varphi}^{0}$ are given functions describing the displacements and the rotations of the plate boundary, respectively. $v$ is the unit outer normal vector to the boundary $S(\boldsymbol{w} \cdot \boldsymbol{n}=0)$. The relations (3) are the static and the kinematic boundary conditions. Other types of boundary conditions are possible. For example, the boundary conditions corresponding to a hinge are given by

$$
v \cdot M \cdot \tau=0, \quad \boldsymbol{u}=0, \quad \boldsymbol{\varphi} \cdot \boldsymbol{\tau}=0 .
$$

Here $\tau$ is the unit tangent vector in the tangential plane to the boundary $S(\pi \cdot n=\tau \cdot \boldsymbol{v}=0)$.

\section{Constitutive Equations}

Polymers near their glass transition temperature behave like viscoelastic materials $[5,6,7,22,23]$. In this case the moduli of the polymers depend on the strain-rate or the time of loading. Thus, a polymeric foam behaves viscoelastically too. Experimental investigations of the vicoelasticity of foams are presented, for example, in [4, 24]. The two-dimensional constitutive equations of a viscoelastic plate were formulated in the general form in [14]. A through-the-thickness symmetric structure of the plate and an isotropic material behavior are considered in [25]. Let us assume here orthotropic material behavior and a plane mid-surface. In this case the two-dimensional viscoelastic constitutive equations for the stress resultants can be formulated as follows:

in-plane forces

$$
T \cdot a=\mathcal{A} \boldsymbol{\mu}+\mathcal{B} \boldsymbol{\kappa} \equiv \int_{-\infty}^{t} \boldsymbol{A}(t-\tau) \cdot \cdot \dot{\boldsymbol{\mu}}(\tau) \mathrm{d} \tau+\int_{-\infty}^{t} \boldsymbol{B}(t-\tau) \cdot \dot{\boldsymbol{\kappa}}(\tau) \mathrm{d} \tau,
$$

transverse shear forces

$$
T \cdot \boldsymbol{n}=\mathcal{G} \gamma \equiv \int_{-\infty}^{t} \Gamma(t-\tau) \cdot \dot{\gamma}(\tau) \mathrm{d} \tau
$$

moments

$$
\boldsymbol{M}^{\mathrm{T}}=\hat{\mathcal{B}} \boldsymbol{\mu}+\mathcal{C} \kappa \equiv \int_{-\infty}^{t} \dot{\boldsymbol{\mu}}(\tau) \cdot \boldsymbol{B}(t-\tau) \mathrm{d} \tau+\int_{-\infty}^{t} \boldsymbol{C}(t-\tau) \cdot \dot{\boldsymbol{\kappa}}(\tau) \mathrm{d} \tau .
$$


Here $\mathcal{A}, \mathcal{B}, \hat{\mathcal{B}}, \mathcal{C}, \mathcal{G}$ are linear viscoelastic operators, $\boldsymbol{A}(t), B(t), C(t)$ are 4 th rank tensors and $\Gamma(t)$ is a 2 nd rank tensor. These tensors describe the effective viscoelastic stiffness properties (relaxation functions for the plate). They depend on the material properties and the cross-section geometry parameters and are given by the relations $[16,19,20,21]$

$$
\begin{aligned}
& A=A_{11} a_{1} a_{1}+A_{12}\left(a_{1} a_{2}+a_{2} a_{1}\right)+A_{22} a_{2} a_{2}+A_{44} a_{4} a_{4}, \\
& B=B_{13} a_{1} a_{3}+B_{14} a_{1} a_{4}+B_{23} a_{2} a_{3}+B_{24} a_{2} a_{4}+B_{42} a_{4} a_{2}, \\
& C=C_{22} a_{2} a_{2}+C_{33} a_{3} a_{3}+C_{34}\left(a_{3} a_{4}+a_{4} a_{3}\right)+C_{44} a_{4} a_{4}, \\
& \Gamma=\Gamma_{1} a_{1}+\Gamma_{2} a_{2}
\end{aligned}
$$

with

$$
a_{1}=a=e_{1} e_{1}+e_{2} e_{2}, \quad a_{2}=e_{1} e_{1}-e_{2} e_{2}, \quad a_{3}=c=e_{1} e_{2}-e_{2} e_{1}, \quad a_{4}=e_{1} e_{2}+e_{2} e_{1} .
$$

$\boldsymbol{e}_{1}, \boldsymbol{e}_{2}$ are unit basic vectors which coincides with the principal directions of the plate.

In the case of isotropic and symmetric over the thickness plates the effective stiffness tensors have the following structure $[16,19,20,21]$

$$
A=A_{11} a_{1} a_{1}+A_{22}\left(a_{2} a_{2}+a_{4} a_{4}\right), \quad C=C_{22}\left(a_{2} a_{2}+a_{4} a_{4}\right)+C_{33} a_{3} a_{3}, \quad \Gamma=\Gamma \boldsymbol{a} .
$$

Using the assumption that $A(t), B(t), C(t)$ and $\Gamma(t)$ do not depend on time with the relations $\boldsymbol{\mu}(-\infty)=\mathbf{0}, \boldsymbol{\kappa}(-\infty)=\mathbf{0}, \boldsymbol{\gamma}(-\infty)=0$ then from Eqs (5) - (7) we obtain the constitutive equations of elastic plates presented in $[16,19,20,21]$.

\section{Viscoelastic Effective Properties}

For elastic plates the identification of the components of the effective stiffness tensors was performed in $[16,19,20,21]$. By the same technique below the analogous viscoelastic stiffness tensor components are computed. Let us consider the three-dimensional viscoelastic constitutive equations $[26,27,28]$

$$
\sigma=\int_{-\infty}^{t} R(t-\tau) \cdot \cdot \dot{\boldsymbol{\varepsilon}}(\tau) \mathrm{d} \tau, \quad \text { or } \quad \boldsymbol{\varepsilon}=\int_{-\infty}^{t} J(t-\tau) \cdot \cdot \dot{\sigma} \mathrm{d} \tau,
$$

where $\sigma$ and $\varepsilon$ are the tensors of stress and strain, $R(t)$ and $J(t)$ are the 4th rank tensors of relaxation and creep functions, respectively.

Further we consider that all properties are functions of the thickness coordinate $z$. That means that in general $\boldsymbol{R}$ and $\boldsymbol{J}$ depend on the thickness coordinate $z$ and on the time $t: \boldsymbol{R}=R(z, t), \boldsymbol{J}=\boldsymbol{J}(z, t)$. In addition, a density function must be considered. Let us assume the simplest case - the density depends only on the thickness coordinate $\rho_{0}=\rho_{0}(z)$, where $\rho_{0}$ is the density of the three-dimensional solid.

Using the Laplace transform

$$
\bar{f}(s)=\int_{0}^{\infty} f(t) e^{-s t} \mathrm{~d} t
$$

one can rewrite Eq. (10) in the form [26, 28]

$$
\bar{\sigma}=s \bar{R}(s) \cdot \bar{\varepsilon}, \quad \bar{\varepsilon}=s \bar{J}(s) \cdot \cdot \bar{\sigma} .
$$

Applying the correspondence principle we can extend the identification procedure $[16,19,20]$ to the Laplace mapping of the effective relaxation or creep functions, see [14]. 
The following non-zero components of the stiffness tensors are valid

$$
\begin{array}{ll}
\bar{A}_{11}=\frac{1}{2}\left\langle\frac{\bar{E}}{1-\bar{v}}\right\rangle, & \bar{A}_{22}=\frac{1}{2}\left\langle\frac{\bar{E}}{1+\bar{v}}\right\rangle=\bar{A}_{44}=\langle\bar{\mu}\rangle, \\
\bar{B}_{13}=-\frac{1}{2}\left\langle\frac{\bar{E}}{1-\bar{v}} z\right\rangle, & \bar{B}_{24}=\frac{1}{2}\left\langle\frac{\bar{E}}{1+\bar{v}} z\right\rangle=-\bar{B}_{42}=\langle\bar{\mu} z>, \\
\bar{C}_{33}=\frac{1}{2}\left\langle\frac{\bar{E}}{1-\bar{v}} z^{2}\right\rangle, & \bar{C}_{44}=\frac{1}{2}\left\langle\frac{\bar{E}}{1+\bar{v}} z^{2}\right\rangle=\bar{C}_{22}=\left\langle\bar{\mu} z^{2}\right\rangle, \\
\bar{\Gamma}_{1}=\bar{\Gamma}=\lambda^{2} \frac{\bar{A}_{44} \bar{C}_{22}-\bar{B}_{42}^{2}}{\bar{A}_{44}}, &
\end{array}
$$

where $\lambda$ is the minimal nonzero eigen-value following from the Sturm-Liouville problem

$$
\frac{\mathrm{d}}{\mathrm{d} z}\left(\bar{\mu} \frac{\mathrm{d} Z}{\mathrm{~d} z}\right)+\lambda^{2} \bar{\mu} \mathrm{Z}=0,\left.\quad \frac{\mathrm{d} Z}{\mathrm{~d} z}\right|_{|z|=\frac{h}{2}}=0 .
$$

Here $\langle\ldots\rangle$ denotes the integration over the plate thickness $h$. For the plate which is symmetrically to the midplane the relation $\boldsymbol{B}=0$ holds true. The relaxation functions of the isotropic viscoelastic plate with symmetric cross-section were considered in [25]. Note that for the isotropic viscoelastic material three functions $\bar{E}(s), \bar{\mu}(s)$ and $\bar{v}(s)$ are introduced. They are interlinked by the formula

$$
\bar{E}=2 \bar{\mu}(1+\bar{v})
$$

Following [29, 30] we use Eq. (14 as the definition of the Poisson's ratio for the viscoelastic material.

In the theory of viscoelasticity of solids the assumption $v(t)=v=$ const is often used. It is fulfilled in many applications (see arguments in [26, 27, 31] concerning $v(t) \approx$ const), for example, $v=0.5$ for an incompressible viscoelastic material. In the general case, $v$ is a function of $t . v(t)$ was considered as an increasing function of $t[22,23,31]$ or non-monotonous function of $t[29,30]$. The latter case may be realized for cellular materials or foams. Further we investigate the influence of $v(t)$ on the deflexion of a viscoelastic plate and its effective relaxation functions.

The tensors of inertia and the plate density are given by $[13,16,21]$

$$
\rho=\left\langle\rho_{0}\right\rangle, \quad \rho \Theta_{1}=-\left\langle\rho_{0} z\right\rangle c, \quad \rho \Theta_{2}=\Theta a, \quad \Theta=\left\langle\rho_{0} z^{2}\right\rangle .
$$

Considering the symmetry in the thickness direction, from (15) one gets that $\boldsymbol{\Theta}_{1}=\mathbf{0}$.

\section{Effective Stiffness Relaxation Functions for FGM}

In this paragraph we consider small deformations of a FGM plate made of a viscoelastic polymer foam. For the panel made from a porous polymer foam the distribution of the pores over the thickness can be inhomogeneous (see, for example, Fig. 1). Let us introduce $h$ as the thickness of the panel, $\rho_{\mathrm{s}}$ as the density of the bulk material and $\rho_{\mathrm{p}}$ as the minimum value of the density of the foam. For the description of the symmetric distribution of the porosity we assume the power law [21]

$$
V(z)=\alpha+(1-\alpha)\left|\frac{2 z}{h}\right|^{n},
$$

where $\alpha=\rho_{\mathrm{p}} / \rho_{\mathrm{s}}$ is the minimal relative density, $n$ is the power.

The properties of the foam strongly depend on the porosity and the cell structure. For the polymer foam in [4] the modification of the standard linear viscoelastic solid is proposed. The constitutive law has the form

$$
\dot{\sigma}+\tau_{\mathrm{E}} \sigma=\kappa(z)\left[E_{\infty} \tau_{\mathrm{E}} \varepsilon+E_{0} \dot{\varepsilon}\right]
$$


where $\kappa(z)=C_{1} V(z)^{2}$ for the open-cell foam and $\kappa(z)=C_{2}\left[\phi^{2} V(z)^{2}+(1-\phi) V(z)\right]$ for the closed-cell foam, respectively. Here $C_{1} \approx 1, C_{2} \approx 1, \phi$ describes the relative volume of the solid polymer concentrated near the cell ribs. Usually, $\phi=0.6 \ldots 0.7 . E_{\infty}, E_{0}, \tau_{\mathrm{E}}$ are material constants of the polymer used in manufacturing of the foam.

From Eq. (16) one can see that the corresponding relaxation function is given by the relations

$$
E=E(z, t)=E(t) \kappa(z), \quad E(t)=E_{\infty}+\left(E_{0}-E_{\infty}\right) e^{-t / \tau_{\mathrm{E}}},
$$

Here $E_{\infty}$ and $E_{0}$ are the equilibrium and the short-time Young's moduli $\left(E_{\infty}<E_{0}\right)$, while $\tau_{\mathrm{E}}$ is the relaxation time for tension. By analogy to (17) the following relation can be established for the shear relaxation function

$$
\mu=\mu(z, t)=\mu(t) m(z) .
$$

Equations (17) and (18) have the meaning that the viscoelastic properties of the foam, for example, the time of relaxation, do not depend on the porosity distribution. Note that the representations (17) and (18) are only rough approximation for spatial nonhomogeneous foams.

Using experimental data presented in $[1,4]$ one can assume $v=$ const. In this case and using Eqs (17), (18) we obtain that $A_{11}, A_{22}, C_{33}, C_{22}$ are related to

$$
A_{11}=\frac{1+v}{1-v} A_{22}, \quad C_{33}=\frac{1+v}{1-v} C_{22}, \quad\left\{A_{22}, C_{22}, \Gamma\right\}=\left\{A_{22}^{\circ}, C_{22}^{\circ}, \lambda^{2} C_{22}^{\circ}\right\} \mu(t) .
$$

For the the closed-cell foam $A_{22}^{\circ}$ and $C_{22}^{\circ}$ are given by

$$
\begin{aligned}
& A_{22}^{\circ}=h\left\{\phi^{2}\left[\alpha^{2}+\frac{2 \alpha(1-\alpha)}{n+1}+\frac{(1-\alpha)^{2}}{2 n+1}\right]+(1-\phi)\left[\alpha+\frac{1-\alpha}{n+1}\right]\right\}, \\
& C_{22}^{\circ}=\frac{h^{3}}{12}\left\{\phi^{2}\left[\alpha^{2}+\frac{6 \alpha(1-\alpha)}{n+3}+\frac{3(1-\alpha)^{2}}{2 n+3}\right]+(1-\phi)\left[\alpha+\frac{3(1-\alpha)}{n+3}\right]\right\} .
\end{aligned}
$$

while for the open-cell foam by Eq. (20) with $\phi=1$. Here we assume that $C_{1}=1, C_{2}=1$, and that $\phi$ does not depend on $z$.

From Eq. (20) it is easy to see that the classical relaxation functions differ only by factors from the shear relaxation function. Note that one can easily extend Eq. (16) to the case of general constitutive equations (10). Thus, using assumption that $v=$ const, one can calculate the classical effective stiffness relaxation functions for general viscoelastic constitutive equations multiplying the shear relaxation function with the corresponding factor similar to Eq. (20). In the more general situation with $v=v(t)$ or taking into account other viscoelastic phenomena, for example, the filtration of a fluid in the saturated foam, the effective stiffness relaxation functions may be more complex then for the pure solid polymer discussed here.

Finally, we should mention that in the case of constant Poisson's ratio and with the assumption (18) the determination of the effective in-plane, bending and transverse shear stiffness tensors of a symmetric FGM viscoelastic plate made of a polymer foam can be realized by the same method as for elastic plates [19, 20, 21]. The relaxation functions for viscoelastic FGM plates can be found from the values of the corresponding effective stiffness of an elastic FGM plate by multiplication with the normalized shear relaxation function of the polymer solid.

\section{Bending of a Viscoelastic Plate composed of FGM}

Let us consider the bending plate problem taking into account viscoelastic material behavior and simply supported boundary conditions. Considering [21, 25], one can reduce Eqs (1) and (2) to one equation for the deflection $w$

$$
s \bar{D}_{\text {eff }} \Delta \Delta \bar{w}=\bar{q}_{n}-\frac{\bar{D}_{\text {eff }}}{\bar{\Gamma}} \Delta \bar{q}_{n^{\prime}}
$$


where $\bar{D}_{\text {eff }}=\bar{C}_{22}+\bar{C}_{33}$ is the Laplace transform of the effective bending stiffness relaxation function. Note that here $\bar{D}_{\text {eff }}=D_{\text {eff }}^{0} \bar{\mu}(s)$, so we obtain that $D_{\text {eff }}=\left(C_{22}^{\circ}+C_{33}^{\circ}\right) \mu(t)$. Assuming that $v=$ const we obtain the relation $\frac{\bar{D}_{\text {eff }}}{\bar{\Gamma}}=\frac{2}{\lambda^{2}(1-v)}$. Looking for the solution of Eq. (21) in the form $w=\sum_{k=1}^{\infty} c_{k}(t) w_{k}(x, y)$, where $w_{k}$ are the eigen-functions satisfying of the equation $\Delta \Delta w_{k}=\omega_{k}^{2} w_{k}$ with simply supported boundary conditions, while $c_{k}$ are unknown functions, we get

$$
\bar{c}_{k}(s)=\frac{1}{s \bar{D}_{\mathrm{eff}} \omega_{k}^{2}}\left(\bar{q}_{n}-\frac{2}{\underline{\lambda^{2}(1-v)}} \Delta \bar{q}_{n}, w_{k}\right), \quad(f, g)=\iint f g d x d y .
$$

The underlined term expresses the difference of this solution from the classical case of the viscoelastic Kirchhoff plate. For the case of a rectangular plate, it was shown in [25] that the maximal deflection may be up to $20 \%$ larger than in the case of the Kirchhoff plate.

\section{Discussion}

The considered approach to model inhomogeneous plates within the framework of a 5-parametric theory of plates has an advantage with respect to the classical theories of sandwich or laminated plates. Further investigations should be directed on the more complex constitutive equations of viscoelastic solids taking into account thermo-mechanical behavior, impact processes and the description of the creep phenomenon in plates made of metal or polymer foams.

\section{References}

[1] M. F. Ashby, A. G. Evans, N. A. Fleck, L. J. Gibson, J. W. Hutchinson and H. N. G. Wadley: Metal Foams: A Design Guid (Butterworth-Heinemann, Boston, 2000)

[2] J. Banhart, M. F. Ashby and N. A. Fleck, editors: Metal Foams and Porous Metal Structures (Verlag MIT Publishing, Bremen, 1999)

[3] H. P. Degischer and B. Kriszt, editors: Handbook of Cellular Metals. Production, Processing, Applications (Wiley-VCH, Weinheim, 2002)

[4] L. J. Gibson and M. F. Ashby: Cellular Solids: Structure and Properties. Cambridge Solid State Science Series (Cambridge University Press, Cambridge, 1997), 2nd edn.

[5] A. H. Landrock, editor: Handbook of Plastic Foams. Types, Properties, Manufactore and Applications (Noes Publications, Park Ridge, New Jersey, 1995)

[6] S. Lee and N. Ramesh, editors: Polymeric Foams. Mechanisms and Materials (CRC Press, Boca Raton, 2004)

[7] N. Mills: Polymer Foams Handbook. Engineering and Biomechanics Applications and Design guide (Butterworth-Heinemann, Amsterdam, 2007)

[8] R. S. Lakes: Science vol. 235, (1987), pp. 1038

[9] M. T. Shaw: Introduction to Polymer Viscoelasticity (Wiley, Hoboken, New Jersey, 2005), 3rd edn.

[10] P. Naghdi: In: Handbuch der Physik, edited by S. Flügge, vol. VIa/2, pp. 425-640 (Springer, 1972) 
[11] E. Reissner: Applied Mechanics Review vol. 38, (1985), pp. 1453

[12] P. A. Zhilin: Int. J. Solids Struct. vol. 12, (1976), pp. 635

[13] H. Altenbach and P. Zhilin: Uspekhi Mekhaniki vol. 11, (1988), pp. 107

[14] H. Altenbach: Ingenieur-Archiv vol. 58, (1988), pp. 215

[15] H. Altenbach and P. A. Zhilin: In: Critical review of the theories of plates and shells and new applications, edited by R. Kienzler, H. Altenbach and I. Ott, Lect. Notes Appl. Comp. Mech. 16, pp. 1-12 (Springer, Berlin, 2004)

[16] P. A. Zhilin: Applied mechanics. Foundations of the theory of shells (in Russ.) (St. Petersburg State Polytechnical University, 2006)

[17] H. Rothert: Direkte Theorie von Linien- und Flächentragwerken bei viskoelastischen Werkstoffverhalten. Techn.-Wiss. Mitteilungen des Instituts für Konstruktiven Ingenieurbaus 73-2, Ruhr-Universität, Bochum (1973)

[18] H. Altenbach: Ingenieur-Archiv vol. 22, (1987), pp. 135

[19] H. Altenbach: Int. J. Solids Struct. vol. 37, (2000), pp. 3503

[20] H. Altenbach: ZAMP vol. 51, (2000), pp. 629

[21] H. Altenbach and V. Eremeyev: Archive of Applied Mechanics vol. DOI:10.1007/s00419-0070192-3

[22] H. F. Brinson and C. L. Brinson: Polymer Engineering Science and Viscoelasticity. An Introduction (Springer, New York, 2008)

[23] E. Riande and et al., editors: Polymer Viscoelasticity: Stress and Strain in Practice (Marcel Dekker, New York, 2000)

[24] A. Kraatz: Berechnung des mechanischen Verhaltens von geschlossenzelligen Schaumstoffen unter Einbeziehung der Mikrostruktur. Diss., zentrum für ingenieurwissenschaften, MartinLuther-Universität, Halle-Wittenberg (2007)

[25] H. Altenbach and V. Eremeyev: ZAMM vol. 88, (2008), pp. 332

[26] R. M. Christensen: Theory of Viscoelasticity. An Introduction (Academic Press, New York, 1971)

[27] A. Drozdov: Finite Elasticity and Viscoelasticity (World Scientific, Singapore, 1996)

[28] P. Haupt: Continuum Mechanics and Theory of Materials (Springer, Berlin, 2002), 2nd edn.

[29] R. S. Lakes: Cellular Polymers vol. 11, (1992), pp. 466

[30] R. S. Lakes and A. Wineman: J. Elasticity vol. 11, (2006), pp. 45

[31] N. Tschoegl: The Phenomenological Theory of Linear Viscoelastic Behavior. An Introduction (Springer, Berlin, 1989) 